\title{
Patterns and predictors of treatment outcome for antenatal major depression
}

\author{
Tze-Ern $\underline{\text { Chua }}^{1,4}$, mBBS, MMed, John Carson $\underline{\text { Allen }}{ }^{2}$, PhD, Loretta $\underline{A n g}^{1}$, MBBS, Li Lian $\underline{O n g}^{1}$, MCounselling, BSocSc, \\ Ying Chia Ch'ng $^{1}$, BBA, MA, Helen $\underline{C h e n}^{1,3,4}$, MBBS, MMed
}

INTRODUCTION Antenatal major depression is a relatively common and potentially debilitating illness, but knowledge of its treatment outcomes and strategies is still lacking. This study aimed to explore the clinical profiles and treatment outcomes of patients with antenatal major depression, to look for patterns and associations that could guide subsequent research and clinical applications.

METHODS From May 2006 to November 2010, 118 consecutive patients with antenatal major depression were naturalistically assessed over eight months of individualised therapy, and their characteristics were assessed as potential predictors of treatment outcome.

RESULTS All participants accepted supportive counselling and case management, although only 51 (43.2\%) participants accepted low-dose antidepressant therapy. Overall, 95 (80.5\%) of them were successfully discharged, while 12 (10.2\%) required extended treatment into the postnatal period. An equation for prognosticating the need for extended treatment was obtained using multiple logistic regression analysis, which incorporated three predictors: previous depression (odds ratio [OR] 12.4, 95\% confidence interval [Cl] 1.40-110; $\mathrm{p}=0.024)$; maternal age $<26$ years or > 35 years (OR 6.88 , $95 \% \mathrm{Cl} 1.67-28.4 ; \mathrm{p}=0.008$ ); and no use of antidepressant (OR 6.94, 95\% Cl 0.79-60.9; $\mathrm{p}=0.080$ ). Among participants with previous depression and at either extreme of maternal age, the number needed to treat with antidepressants to avert extended treatment was three.

CONCLUSION The majority of women with antenatal major depression recovered after receiving short-term treatment. Those with previous depression and who were of relative extreme maternal age were most likely to benefit from antidepressant treatment to expedite recovery.

Keywords: antenatal, antidepressant, major depression, psychiatric case management, treatment outcome

\section{INTRODUCTION}

Major depression affects one in 14 women in Singapore, and with a lifetime risk of $5.4 \%$ to $8.6 \%$ for women aged $18-49$ years, ${ }^{(1)}$ major depression may occur during pregnancy. Given the nation's live-birth rate of 42,185 in $2015,{ }^{(2)}$ in conjunction with local hospital-based prevalence rates of $11 \%{ }^{(3)}$ to $12.5 \%{ }^{(4)}$ for antenatal major depression, there may well be several thousand patients per year.

The impact of antenatal major depression has been firmly established through clinical research, particularly over the past decade. The presence of antenatal major depression has been shown to increase the odds of maternal suicidal ideation by a factor of 11.5. ${ }^{(5)}$ In addition, it is associated with impaired fetal growth and prematurity, ${ }^{(6)}$ and is a major risk factor for postnatal depression. ${ }^{(7)}$ Local studies show that antenatal major depression is linked to smaller birth size ${ }^{(8)}$ and structural brain changes ${ }^{(9)}$ in neonates. As toddlers, children of depressed mothers have more emotional and behavioural difficulties and poorer verbal intelligence than children of non-depressed mothers. ${ }^{(10)}$

Unfortunately, little is known about the treatment strategies and medications for antenatal major depression. A meta-analysis of 27 studies showed that psychological treatments for perinatal depression have moderate efficacy, and pharmacological trials are difficult to analyse meaningfully, ${ }^{(11)}$ as they are in the minority, possibly due to the ethical limitations of studying medication use during pregnancy. The bulk of research on antenatal antidepressant use focuses on fetal side effects, such as cardiac malformations, persistent pulmonary hypertension of the newborn (PPHN) and neonatal poor adaptation syndrome. These studies tend to be small-scale, retrospective and/or varied in methodology and results, ${ }^{(12)}$ and some do not factor in the impact of untreated maternal depression. ${ }^{(13)}$ With these points taken into account, recent reviews posit that antenatal antidepressant use may be less risky than previously thought, but more research is necessary to clarify this assumption. ${ }^{(14,15)}$

A second limitation to studying antenatal major depression is the lack of a validated rating scale for specifically measuring symptom progression throughout pregnancy. Trials that employ the Edinburgh Postnatal Depression Scale, Beck Depression Inventory and Hamilton Rating Scale for Depression have shown that optimal cut-off scores fluctuate when they are administered sequentially, and according to trimester, gravidity and learning effect. ${ }^{(16)}$ In the absence of a reliable measure, it is difficult to reach a definitive consensus about the efficacy of antidepressants in pregnancy.

A third obstacle to managing antenatal major depression is that clinicians lack definable indicators to identify patients who would benefit most from the targeted use of antidepressants

\footnotetext{
${ }^{1}$ Department of Psychological Medicine, KK Women's and Children's Hospital, ${ }^{2}$ Office of Clinical Sciences - Centre for Quantitative Medicine, Duke-NUS Medical School, ${ }^{3}$ Postnatal Depression Intervention Programme, KK Women's and Children's Hospital, ${ }^{4}$ Duke-NUS Medical School, Singapore

Correspondence: Dr Chua Tze-Ern, Senior Consultant, Department of Psychological Medicine, KK Women's and Children's Hospital, 100 Bukit Timah Road, Singapore 229899. chua.tze.ern@singhealth.com.sg
} 
while avoiding unnecessary side effects, and worry for those who would not benefit from the treatment. Predictors of onset of antenatal major depression have been identified to some extent, but little is known regarding the predictors of treatment outcome. These knowledge deficits may impede the appropriate use of pharmacotherapy, as well as psychoeducation and prognostication.

Despite the various knowledge gaps, there is evidence to guide the management of antenatal major depression. Sertraline has been indicated as a pharmacological option, as it shows less placental transfer than fluoxetine, ${ }^{(13,17)}$ with low risks of major congenital anomalies and neonatal poor adaptation syndrome. Tricyclic antidepressants, such as dothiepin, do not impair offspring neurodevelopment ${ }^{(14)}$ and can be used at low doses with demonstrable efficacy and few side effects. ${ }^{(18)}$ Other studies suggest general practices, such as avoiding medication in the first trimester to suppress the risk of teratogenicity, avoiding selective serotonin reuptake inhibitors (SSRIs) in late pregnancy to help avert PPHN, ${ }^{(13)}$ and using the lowest possible dose, since antidepressants as a class may increase the risk of prematurity. ${ }^{(19)}$

With the aid of what is known and despite all that remains unknown, our clinical experience with antenatal major depression continues to expand and develop, contributing to greater knowledge and more effective practice. We herein present the treatment outcomes of patients with antenatal major depression who were managed during our first five years as a psychiatric service in Singapore's largest maternity unit, while focusing on identifying predictors of poor clinical outcome.

\section{METHODS}

With approval from the institutional review board, clinical data of eligible patients under the care of the perinatal psychiatric service at KK Women's and Children's Hospital (KKH), Singapore, was reviewed. This is a liaison service that was started in 2006. It receives referrals, from within and outside the hospital, of female patients requiring pregnancy-related mental healthcare, most commonly for depressive and anxiety disorders, but also for psychotic and pre-existing psychiatric disorders. These patients receive multidisciplinary care from psychiatrists who have a special interest in perinatal mental health and from psychiatric case managers. Treatment options include medications that are suitable for perinatal use, counselling and case management comprising personalised therapeutic engagement, psychoeducation and resource linkage. ${ }^{(20)}$

The participants in this study consisted of pregnant women outpatients with a primary diagnosis of antenatal major depression made by a board-certified psychiatrist. They met the criteria for having a major depressive episode, as defined by the fourth edition of the Diagnostic and Statistical Manual of Mental Disorders, occurring at any time during pregnancy. Those who had concurrent secondary diagnoses, such as mild anxiety symptoms or personality disorders, were also eligible for participation in the study. All 118 eligible patients seen from May 2006 to November 2010 agreed to participate in the study. Patients who had milder variants of depression, such as adjustment reaction or adjustment disorder, or other primary psychiatric diagnoses, such as bipolar disorder or schizophrenia, were excluded from the study.

Participant data was obtained under informed consent and systematically recorded during baseline visits. In addition to sociodemographic details (e.g. ethnicity, age and occupation), information on psychiatric and obstetric backgrounds, including known predictors of illness onset (e.g. previous depression, young maternal age, unplanned pregnancy and previous abuse), was also gathered. ${ }^{(21-23)}$

Following clinical diagnosis, antidepressant medication was offered to participants, who were also counselled on its use. As some women had concerns regarding the fetal side effects of antidepressant therapy, only participants who gave consent were administered the medication. Options of antidepressant medication included sertraline (up to $75 \mathrm{mg}$ daily) for participants who preferred less-sedating medication and dothiepin (up to $75 \mathrm{mg}$ daily) for participants who required sedative aid for sleep. First-trimester use was avoided, and clinicians monitored treatment compliance and side effects at each participant's follow-up session.

A psychiatric case manager was assigned to each participant to build therapeutic rapport and maintain regular contact via telephone calls between clinical visits. The case managers also provided individualised care through psychoeducation, emotional support, counselling and encouraging compliance with treatment, as often as required or preferred by the participant. Outpatient sessions ranged in frequency, from 1-2 weeks for severely ill participants to 4-6 weeks for those with stable condition.

The proportion of participants who were discharged from follow-up in comparison with those who remained in treatment began to plateau after seven months of treatment. In view of that, we used a follow-up period of eight months to give a margin of allowance, after which time all participants would have entered the postnatal phase. We defined participants' treatment outcomes as: (a) discharged from follow-up in stable condition; (b) required extended treatment for the current episode; and (c) defaulted or transferred follow-up eight months after their first clinical visit. To ensure that participants were correctly categorised, the case managers made telephone contact to verify that those in the first category were well and that those in the third category had elected to discontinue follow-up at our centre.

Fisher's exact test was used to compare frequency distributions of categorical baseline characteristics between participants who were discharged in stable condition and those who required extended treatment, and between participants who completed the eight-month follow-up and those who defaulted. Univariate and multivariate logistic regression analyses were used to assess participant characteristics as potential predictors of treatment outcome. Univariate logistic regression analysis was performed for each variable and a crude (unadjusted) odds ratio (OR) with 95\% confidence interval $(\mathrm{Cl})$ obtained.

To reduce the risk of making a Type II error (i.e. failure to detect an effect that is present) in this exploratory study, variables that were significant at $p \leq 0.20$ on univariate analysis were subjected to two multivariate analysis approaches: (a) an 
analysis in which all variables were entered into the model; and (b) a variable selection algorithm incorporating likelihood ratio backward elimination with significance level to stay $\leq 0.10$ to develop a parsimonious predictive model. The p-values for significance levels were obtained using the Wald chi-square statistical test. Predicted probabilities of clinical outcomes were calculated from the fitted logistic regression model. Receiver operating characteristic (ROC) analysis was used to assess the overall predictive accuracy of the final logistic regression model. Unless otherwise specified, statistical significance was set at $\mathrm{p} \leq 0.05$ for all analyses. All calculations were performed using IBM SPSS Statistics version 19.0 (IBM Corp, Armonk, NY, USA).

\section{RESULTS}

We evaluated 118 consecutive patients with antenatal major depression and analysed them according to the treatment outcomes: (a) 95 (80.5\%; Clopper-Pearson 95\% Cl 72.2\%-87.2\%) patients were discharged in stable condition after eight months ('discharged'); (b) 12 (10.2\%) patients required treatment that extended into the postnatal period ('extended treatment'); and (c) $11(9.3 \%)$ patients did not complete the duration of the study follow-up ('defaulted'; Table I).

Among patients who completed the study ('completers'; $\mathrm{n}=107$ ), those who declined antidepressant medication did so out of concern that it would cause fetal side effects. The 11

Table I. Comparison of characteristics between patients who were discharged versus those requiring extended treatment, and between all patients versus those who defaulted.

\begin{tabular}{|c|c|c|c|c|c|c|}
\hline \multirow[t]{3}{*}{ Characteristic } & \multicolumn{6}{|c|}{ No. (\%) } \\
\hline & \multicolumn{3}{|c|}{ Completed $(n=107)$} & \multicolumn{3}{|c|}{ All participants $(n=118)$} \\
\hline & $\begin{array}{l}\text { Discharged } \\
\quad(n=95)\end{array}$ & $\begin{array}{c}\text { Extended } \\
\text { treatment }(n=12)\end{array}$ & p-value & $\begin{array}{l}\text { Completed } \\
(n=107)\end{array}$ & $\begin{array}{l}\text { Defaulted } \\
(n=11)\end{array}$ & p-value \\
\hline Ethnicity & & & 0.301 & & & 0.099 \\
\hline Chinese & $62(65.3)$ & $11(91.7)$ & & $73(68.2)$ & $7(63.6)$ & \\
\hline Malay & $18(18.9)$ & $1(8.3)$ & & $19(17.8)$ & $0(0)$ & \\
\hline Indian & $7(7.4)$ & $0(0)$ & & $7(6.5)$ & $1(9.1)$ & \\
\hline Other & $8(8.4)$ & $0(0)$ & & $8(7.5)$ & $3(27.3)$ & \\
\hline Age (yr) & & & $0.044^{*}$ & & & 0.495 \\
\hline$\leq 20$ & $5(5.3)$ & $1(8.3)$ & & $6(5.6)$ & $0(0)$ & \\
\hline $21-25$ & $13(13.7)$ & $3(25.0)$ & & $16(15.0)$ & $1(9.1)$ & \\
\hline $26-30$ & $36(37.9)$ & $3(25.0)$ & & $39(36.4)$ & $7(63.6)$ & \\
\hline $31-35$ & $33(34.7)$ & $1(8.3)$ & & $34(31.8)$ & $2(18.2)$ & \\
\hline$\geq 36$ & $8(8.4)$ & $4(33.3)$ & & $12(11.2)$ & $1(9.1)$ & \\
\hline Marital status & & & $0.037^{*}$ & & & 0.071 \\
\hline Married & $92(96.8)$ & $10(83.3)$ & & $102(95.3)$ & $9(81.8)$ & \\
\hline Unmarried & $3(3.2)$ & $2(16.7)$ & & $5(4.7)$ & $2(18.2)$ & \\
\hline Current episode onset & & & 0.493 & & & 0.882 \\
\hline Pre-pregnancy & $23(24.2)$ & $4(33.3)$ & & $27(25.2)$ & $3(27.3)$ & \\
\hline During pregnancy & $72(75.8)$ & $8(66.7)$ & & $80(74.8)$ & $8(72.7)$ & \\
\hline Previous depression & & & $0.005^{*}$ & & & $0.033^{*}$ \\
\hline Perinatal & $23(24.2)$ & $8(66.7)$ & & $31(29.0)$ & $2(18.2)$ & \\
\hline Non-perinatal & $25(26.3)$ & $3(25.0)$ & & $28(26.2)$ & $7(63.6)$ & \\
\hline None & $47(49.5)$ & $1(8.3)$ & & $48(44.9)$ & $2(18.2)$ & \\
\hline $\begin{array}{l}\text { Other psychiatric } \\
\text { illness }\end{array}$ & & & 0.164 & & & 0.424 \\
\hline Present & $22(23.2)$ & $5(41.7)$ & & $27(25.2)$ & $4(36.4)$ & \\
\hline Absent & $73(76.8)$ & $7(58.3)$ & & $80(74.8)$ & $7(63.6)$ & \\
\hline Familial depression & & & 0.981 & & & 0.296 \\
\hline Present & $32(33.7)$ & $4(33.3)$ & & $36(33.6)$ & $2(18.2)$ & \\
\hline Absent & $63(66.3)$ & $8(66.7)$ & & $71(66.4)$ & $9(81.8)$ & \\
\hline Planned pregnancy & & & 0.394 & & & 0.539 \\
\hline Planned & $44(46.3)$ & $4(33.3)$ & & $48(44.9)$ & $6(54.5)$ & \\
\hline Unplanned & $51(53.7)$ & $8(66.7)$ & & $59(55.1)$ & $5(45.5)$ & \\
\hline Abortion(s) & & & 0.365 & & & 0.127 \\
\hline Present & $18(18.9)$ & $1(8.3)$ & & $19(17.8)$ & $0(0)$ & \\
\hline Absent & $77(81.1)$ & $11(91.7)$ & & $88(82.2)$ & $11(100.0)$ & \\
\hline
\end{tabular}




\begin{tabular}{|c|c|c|c|c|c|c|}
\hline \multirow[t]{3}{*}{ Characteristic } & \multicolumn{6}{|c|}{ No. (\%) } \\
\hline & \multicolumn{3}{|c|}{ Completed $(n=107)$} & \multicolumn{3}{|c|}{ All participants $(n=118)$} \\
\hline & $\begin{array}{l}\text { Discharged } \\
(n=95)\end{array}$ & $\begin{array}{c}\text { Extended } \\
\text { treatment }(n=12)\end{array}$ & $\mathrm{p}$-value & $\begin{array}{l}\text { Completed } \\
(n=107)\end{array}$ & $\begin{array}{l}\text { Defaulted } \\
(n=11)\end{array}$ & p-value \\
\hline Miscarriage(s) & & & 0.172 & & & 0.220 \\
\hline Present & $13(13.7)$ & $0(0)$ & & $13(12.1)$ & $0(0)$ & \\
\hline Absent & $82(86.3)$ & $12(100.0)$ & & $94(87.9)$ & $11(100.0)$ & \\
\hline Physical illness & & & 0.495 & & & 0.598 \\
\hline Present & $15(15.8)$ & $1(8.3)$ & & $16(15.0)$ & $1(9.1)$ & \\
\hline Absent & $80(84.2)$ & $11(91.7)$ & & $91(85.0)$ & $10(90.9)$ & \\
\hline Previous abuse & & & $0.037^{*}$ & & & 0.464 \\
\hline Present & $3(3.2)$ & $2(16.7)$ & & $5(4.7)$ & $0(0)$ & \\
\hline Absent & $92(96.8)$ & $10(83.3)$ & & $102(95.3)$ & $11(100.0)$ & \\
\hline Occupation & & & $0.017^{*}$ & & & 0.746 \\
\hline Not employed & $38(40.0)$ & $3(25.0)$ & & $41(38.3)$ & $5(45.5)$ & \\
\hline Professional & $27(28.4)$ & $3(25.0)$ & & $30(28.0)$ & $2(18.2)$ & \\
\hline Executive & $4(4.2)$ & $0(0)$ & & $4(3.7)$ & $0(0)$ & \\
\hline Administrative & $16(16.8)$ & $1(8.3)$ & & 17 (15.9) & $1(9.1)$ & \\
\hline Service & $10(10.5)$ & 4 (33.3) & & $14(13.1)$ & $3(27.3)$ & \\
\hline Self-employed & $0(0)$ & $1(8.3)$ & & $1(0.9)$ & $0(0)$ & \\
\hline Cigarette use & & & 0.300 & & & 0.071 \\
\hline Present & $13(13.7)$ & $3(25.0)$ & & $16(15.0)$ & $4(36.4)$ & \\
\hline Absent & $82(86.3)$ & $9(75.0)$ & & $91(85.0)$ & $7(63.6)$ & \\
\hline Alcohol use & & & 0.218 & & & 0.273 \\
\hline Present & $2(2.1)$ & $1(8.3)$ & & $3(2.8)$ & $1(9.1)$ & \\
\hline Absent & $93(97.9)$ & $11(91.7)$ & & $104(97.2)$ & $10(90.9)$ & \\
\hline Drug use & & & 0.218 & & & 0.574 \\
\hline Present & $2(2.1)$ & $1(8.3)$ & & $3(2.8)$ & $0(0)$ & \\
\hline Absent & 93 (97.9) & $11(91.7)$ & & $104(97.2)$ & $11(100.0)$ & \\
\hline Antidepressant therapy & & & $0.012^{*}$ & & & 0.426 \\
\hline Accepted & $44(46.3)$ & $1(8.3)$ & & $45(42.1)$ & $6(54.5)$ & \\
\hline Declined & $51(53.7)$ & $11(91.7)$ & & $62(57.9)$ & $5(45.5)$ & \\
\hline
\end{tabular}

${ }^{*} \mathrm{p} \leq 0.05$ was statistically significant

defaulters differed from the 107 completers only with regard to previous non-perinatal depression, the presence of which increased the odds of defaulting treatment to $4.94(95 \% \mathrm{Cl}$ 1.34-18.2; $p=0.015)$.

After being dichotomised, the characteristics of the 107 completers were subjected to univariate logistic regression analysis to obtain the crude OR for each variable, to determine if it was a risk factor for requiring extended treatment. Variables that were significant at $p \leq 0.20$ in this univariate analysis were: previous depression; no antidepressant therapy; age $<26$ years or > 35 years; service job; being unmarried; previous abuse; Chinese ethnicity; and other psychiatric illness. These eight variables were subjected to multivariate logistic regression analysis: (a) as a block; and then (b) to a backward elimination selection algorithm to obtain a final parsimonious predictive model for requiring extended treatment versus being discharged in stable condition (Table II).

The final three-factor model, comprising previous depression, antidepressant therapy and age, yielded R2 $=36.9 \%$ (Nagelkerke) and showed no lack of fit with the observed frequency distribution (Hosmer-Lemeshow $\chi^{2}(5)=1.716 ; p=0.887$ ). Area under the ROC curve (AUC) was 0.861 (95\% Cl 0.761-0.960; $\mathrm{p}<0.001)$. A prognostic cut-point based on a predicted probability of 0.323 resulted in $58.3 \%$ sensitivity and $93.7 \%$ specificity. Given that the prevalence rate for extended treatment was $10.2 \%$ (12/118 patients), the positive and negative predictive values for this model and cut-point were $51.2 \%$ and $95.2 \%$, respectively. Predicted probabilities for requiring extended treatment were calculated using the fitted model for all possible combinations of the variables: previous depression, antidepressant therapy and age (Fig. 1).

Among patients with previous depression, the number needed to treat with antidepressants to avert extended treatment was three for those who were aged $<26$ years or $>35$ years and nine for those who were aged $26-35$ years. Among patients with no previous depression, the number needed to treat with antidepressants was 15 for those who were aged $<26$ years or $>35$ years and 96 for those who were aged 26-35 years.

\section{DISCUSSION}

To the best of our knowledge, this is the first study to examine treatment outcome for antenatal major depression among 
Table II. Univariate and multivariate logistic regression analyses of patient characteristics as predictors for patients requiring extended treatment.

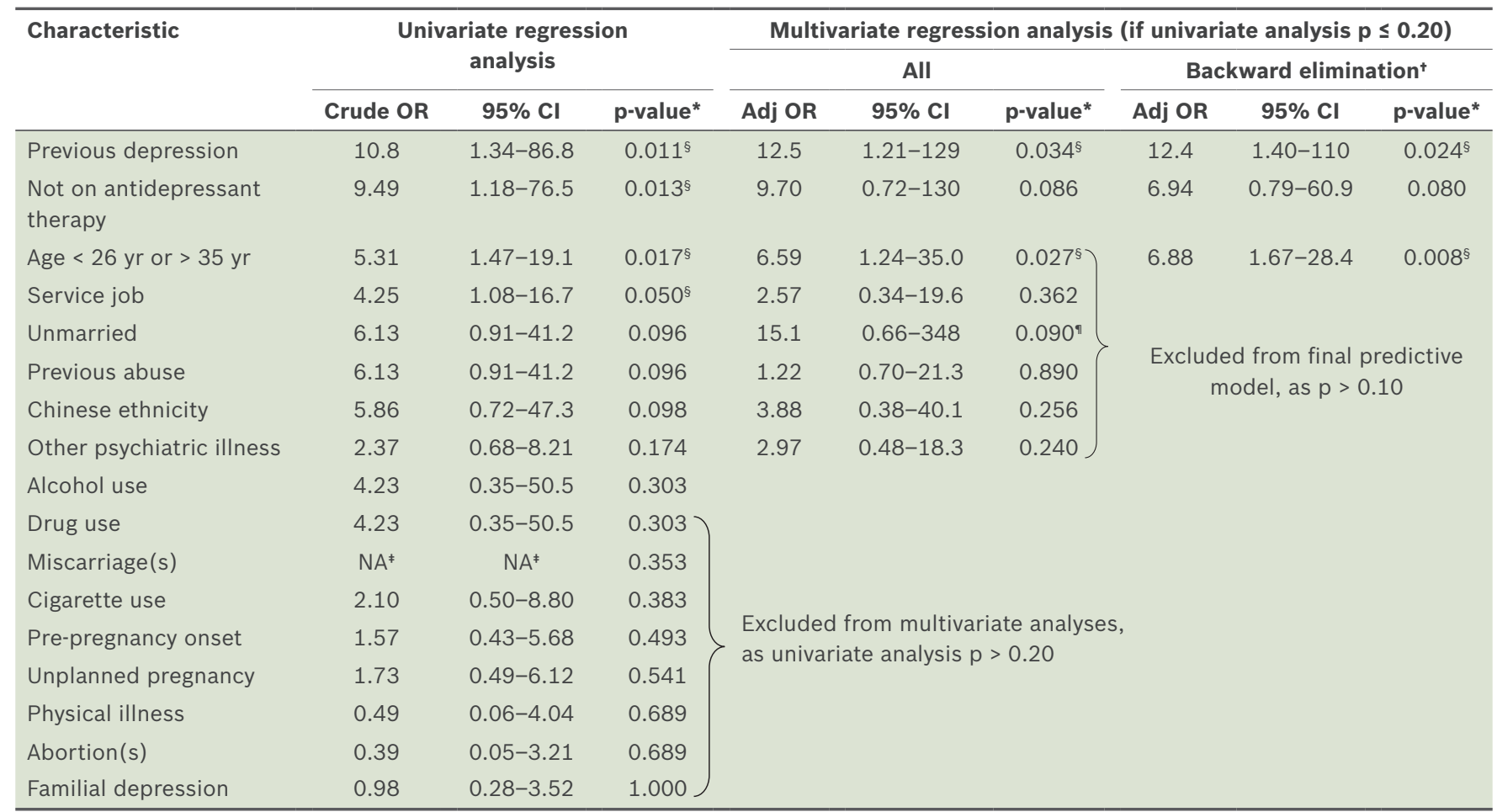

${ }^{*}$ Calculated using Wald chi-square test. +Based on likelihood ratio stepwise backward elimination with $\mathrm{SLS} \leq 0.10$. $¥ \mathrm{As}$ one cell count $=0$. $\S p \leq 0.05$ was statistically significant. I p = 0.115 upon backward elimination regression and therefore excluded from the final model. Adj: adjusted; Cl: confidence interval; NA: not applicable; OR: odds ratio; SLS: significance level to stay

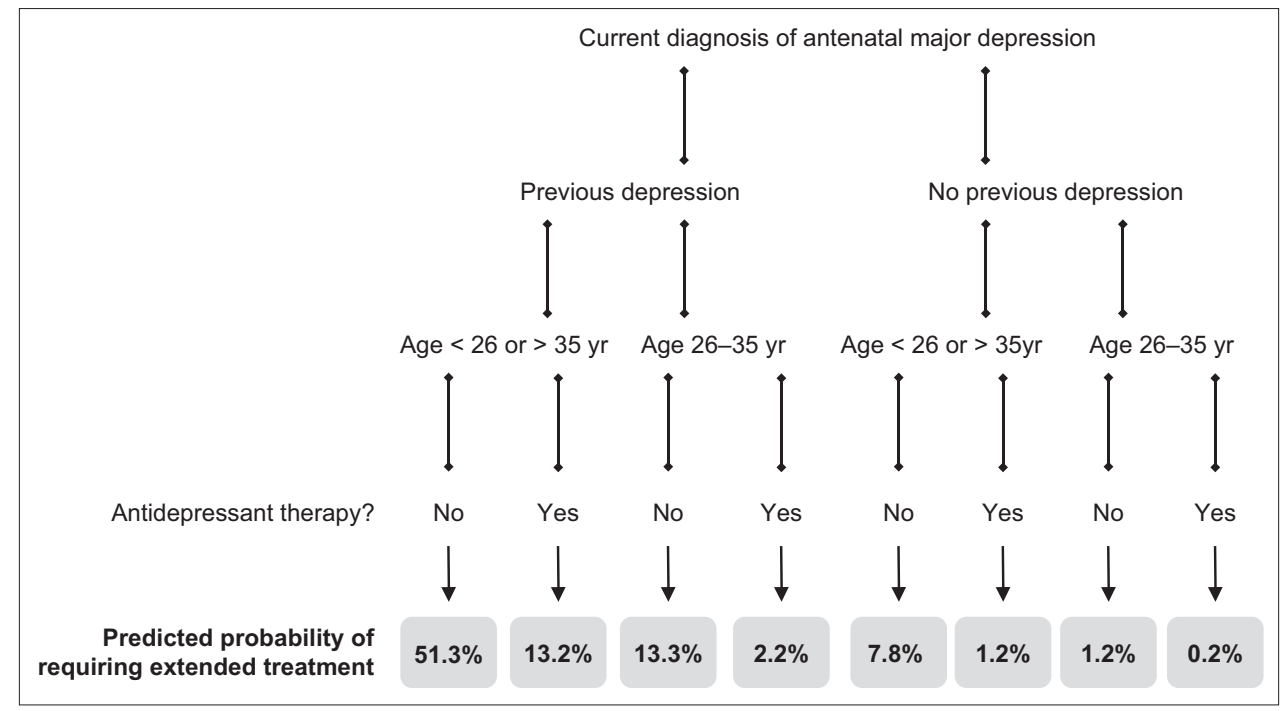

Fig. 1 Flowchart shows the predicted probabilities of requiring extended treatment for antenatal major depression.

women in Singapore. Among the variables investigated, we found that the three most highly prognostic variables for whether extended psychiatric treatment would be needed were previous depression, relative extremes of maternal age and acceptance of antidepressant use; specifically, antidepressant use exerted a differential clinical benefit for reducing the need for extended treatment, which was greatest in previously depressed patients at either extreme of maternal age. Although our analysis focused primarily on identifying this model, our results also indicated that $88.8 \%$ of patients could expect to be successfully discharged within eight months, with an even higher proportion in first-episode cases (47/48 patients, 97.9\%).

Our findings are supported by existing evidence, such as a longitudinal study that showed a recovery rate of $69 \%$ in the absence of previous postnatal depression compared with $20 \%$ when depression is present. ${ }^{(24)}$ Patients with repeated depressive episodes, and women in particular, ${ }^{(25)}$ become increasingly sensitised to stress ${ }^{(26)}$ and experience increasingly frequent recurrences. ${ }^{(27)}$ A possible explanation is a link between depression and hypothalamic-pituitary-adrenal axis 
dysregulation. ${ }^{(28)}$ This leads to hippocampal atrophy ${ }^{(29,30)}$ and cognitive impairment with prolongation of mood symptoms ${ }^{(31)}$ that appear to worsen with recurrent depression. ${ }^{(32)}$ Fortunately, there is concurrent evidence that antidepressants are neuroprotective. SSRIs, such as sertraline, ${ }^{(33)}$ fluoxetine and escitalopram, ${ }^{(34)}$ have been shown to upregulate hippocampal neurogenesis in animal brains and in vitro human progenitor cells, thereby reversing the biological impact of depression.

It has also been observed that extremes of maternal age are linked to postnatal depression, ${ }^{(7)}$ adverse obstetric outcomes, and metabolic syndrome and fetal anomalies in older mothers. ${ }^{(35)}$ The prolongation of antenatal major depression in these women may be related to the need to cope with poorer physical health and higher obstetric risks, as well as the social impact of being pregnant at their age. It is still unknown whether biological factors are also involved.

Another point of interest is the small number of participants available to our study. KKH sees over 14,000 births per year, ${ }^{(36)}$ and applying a conservative prevalence estimate of $11 \%$ for antenatal major depression translates to 1,540 patients per year. In this context, our study cohort of 118 consecutive patients over five years represents only a small fraction of the actual cases, reflecting the low rate of help-seeking for psychiatric illness. In Singapore, it has been observed that less than a third of individuals with depression or anxiety acknowledge that they have mental health problems, ${ }^{(37)}$ and only $5.9 \%$ of those who screen positive for psychiatric illness had sought professional help. ${ }^{(38)}$ Perinatal depression is also associated with other specific barriers to help-seeking, such as the expectation of being able to cope well with motherhood, time constraints and limited healthcare access. ${ }^{(39,40)}$

Although reasonably congruous with the existing literature, our findings are more exploratory than definitive, as they were based on a modest number of patients receiving open-label treatment. At the time of initiation of this psychiatric service, we did not know how many patients would seek help for antenatal major depression, the treatment options they would accept, or the expected range of therapeutic response in an Asian population, and so we opted for a naturalistic observation of standard clinical practice in this study. Our results are therefore limited by non-qualitative assessments, recall bias, inter-rater variability and wide Cls.

Non-pharmacological options still remain the mainstay of perinatal psychiatric care. Similar future studies may be improved by the addition of neonatal outcome, psychosocial stress and personality trait measures, but may still be hampered by small participant numbers or the lack of a dedicated rating scale for monitoring antenatal depression over time. The present study provides useful data for planning subsequent studies that incorporate more rigorous quantitative methods.

Limitations notwithstanding, our study exhibited strengths that included its range of considered sociodemographic and clinical factors, assessments made by board-certified psychiatrists and a statistically significant prognostic model for extended treatment based on readily obtainable clinical data $(A \cup C=0.861)$.
The default rate was relatively low (9.3\% over eight months) and the defaulters differed significantly from the completers only in having a higher prevalence of previous non-perinatal depression, suggesting a reversion to pre-existing psychiatric care. Nonetheless, even among participants with previous non-perinatal depression, $80.0 \%$ (28/35) remained in our care for the duration of the study.

In conclusion, our early experience in perinatal psychiatry indicates that most women recover from antenatal major depression after short-term treatment, particularly if they have not been previously depressed and are in their mid-twenties to mid-thirties. For women with previous depression and at relative extremes of maternal age who are more likely to require longer-term psychiatric care, antidepressant therapy may expedite their recovery. We hope that our findings will reduce the stigma of antenatal major depression and encourage affected women to seek medical help, and also contribute to the development of clinical therapeutic algorithms in which antidepressants could be preferentially recommended to patients who are most likely to benefit, but are otherwise used sparingly. This strategy would help clinicians obtain optimal benefit with minimal risk from this controversial treatment option. Further evaluation of targeted treatment strategies for specific antenatal subgroups may yield more safe and effective ways of giving depressed women a better start to motherhood.

\section{REFERENCES}

1. Chong SA, Abdin E, Vaingankar JA, et al. A population-based survey of mental disorders in Singapore. Ann Acad Med Singapore 2012; 41:49-66.

2. Department of Statistics, Singapore. Births and deaths [updated 23 May 2016]. Available at: http://www.singstat.gov.sg/statistics/latest-data\#16. Accessed May $24,2016$.

3. Thiagayson P, Krishnaswamy G, Lim ML, et al. Depression and anxiety in Singaporean high-risk pregnancies - prevalence and screening. Gen Hosp Psychiatry 2013; 35:112-6.

4. Keleher V, Nyunt SZ, Broekman B, Chee C. Prevalence and risk factors for antenatal depression in pregnant women attending National University Hospital, Singapore. Eur Psychiatry 2012; 27 Suppl 1:1.

5. Gavin AR, Tabb KM, Melville JL, Guo Y, Katon W. Prevalence and correlates of suicidal ideation during pregnancy. Arch Womens Ment Health 2011; 14:239-46.

6. Field T, Diego M, Hernandez-Reif M. Prenatal depression effects on the fetus and newborn: a review. Infant Behav Dev 2006; 29:445-55.

7. Milgrom J, Gemmill AW, Bilszta JL, et al. Antenatal risk factors for postnatal depression: a large prospective study. J Affect Disord 2008; 108:147-57.

8. Broekman BF, Chan YH, Chong YS, et al; GUSTO Research Group. The influence of anxiety and depressive symptoms during pregnancy on birth size. Paediatr Perinat Epidemiol 2014; 28:116-26.

9. Rifkin-Graboi A, Bai J, Chen $\mathrm{H}$, et al. Prenatal maternal depression associates with microstructure of right amygdala in neonates at birth. Biol Psychiatry 2013; 74:837-44.

10. Barker ED, Jaffee SR, Uher R, Maughan B. The contribution of prenatal and postnatal maternal anxiety and depression to child maladjustment. Depress Anxiety $2011 ; 28: 696-702$.

11. Sockol LE, Epperson CN, Barber JP. A meta-analysis of treatments for perinatal depression. Clin Psychol Rev 2011; 31:839-49.

12. Gentile $S$, Bellantuono C. Selective serotonin reuptake inhibitor exposure during early pregnancy and the risk of fetal major malformations: focus on paroxetine. J Clin Psychiatry 2009; 70:414-22.

13. Byatt N, Deligiannidis KM, Freeman MP. Antidepressant use in pregnancy: a critical review focused on risks and controversies. Acta Psychiatr Scand 2013; 127:94-114.

14. Gentile S, Galbally M. Prenatal exposure to antidepressant medications and neurodevelopmental outcomes: a systematic review. J Affect Disord 2011; 128:1-9.

15. Koren G, Nordeng HM. Selective serotonin reuptake inhibitors and malformations: case closed? Semin Fetal Neonatal Med 2013; 18:19-22. 
16. Ji S, Long Q, Newport DJ, et al. Validity of depression rating scales during pregnancy and the postpartum period: impact of trimester and parity. J Psychiat Res 2011; 45:213-9.

17. Hendrick V, Stowe ZN, Altshuler LL, et al. Placental passage of antidepressant medications. Am J Psychiatry 2003; 160:993-6.

18. Furukawa TA, McGuire H, Barbui C. Meta-analysis of effects and side effects of low dosage tricyclic antidepressants in depression: systematic review. BM] $2002 ; 325: 991$.

19. Huang H, Coleman S, Bridge JA, Yonkers K, Katon W. A meta-analysis of the relationship between antidepressant use in pregnancy and the risk of preterm birth and low birth weight. Gen Hosp Psychiatry 2014; 36:13-8.

20. Ch'ng YC, Wang J, Chen H. Perinatal case management - caring for mothers as they care for babies. Best Pract Res Clin Obstet Gynaecol 2010; 227-32.

21. Bunevicius R, Kusminskas L, Bunevicius A, et al. Psychosocial risk factors for depression during pregnancy. Acta Obstet Gynecol Scand 2009; 88:599-605.

22. Rich-Edwards JW, Kleinman K, Abrams A, et al. Sociodemographic predictors of antenatal and postpartum depressive symptoms among women in a medical group practice. J Epidemiol Community Health 2006; 60:221-7.

23. Leigh B, Milgrom J. Risk factors for antenatal depression, postnatal depression and parenting stress. BMC Psychiatry 2008; 8:24.

24. Sexton MB, Flynn HA, Lancaster C, et al. Predictors of recovery from prenatal depressive symptoms from pregnancy through postpartum. J Womens Health (Larchmt) 2012; 21:43-9.

25. You S, Conner KR. Stressful life events and depressive symptoms: influences of gender, event severity, and depression history. J Nerv Ment Dis 2009; 197:829-33.

26. Post RM. Transduction of psychosocial stress into the neurobiology. Am J Psychiatry 1992; 149:999-1010.

27. Kessing LV, Hansen MG, Andersen PK, Angst J. The predictive effect of episodes on the risk of recurrence in depressive and bipolar disorders - a life-long perspective. Acta Psychiatr Scand 2004; 109:339-44.

28. Brummelte S, Galea LA. Depression during pregnancy and postpartum contribution of stress and ovarian hormones. Prog Neuropsychopharmacol Biol Psychiatry 2010; 34:766-76.

29. Frodl T, O'Keane V. How does the brain deal with cumulative stress? A review with focus on developmental stress, HPA axis function and hippocampal structure in humans. Neurobiol Dis 2013; 52:24-37.

30. Sheline YI, Gado MH, Kraemer HC. Untreated depression and hippocampal volume loss. Am J Psychiatry 2003; 160:1516-8.

31. Gradin VB, Pomi A. The role of hippocampal atrophy in depression: a neurocomputational approach. J Biol Phys 2008; 34:107-20.

32. Sheline YI, Wang PW, Gado MH, Csernansky JG, Vannier MW. Hippocampal atrophy in recurrent major depression. Proc Natl Acad Sci U S A 1996; 93:3908-13.

33. Anacker C, Zunszain PA, Cattaneo A, et al. Antidepressants increase human hippocampal neurogenesis by activating the glucocorticoid receptor. Mol Psychiatry 2011; 16:738-50.

34. Hanson ND, Owens MJ, Nemeroff CB. Depression, antidepressants, and neurogenesis: a critical reappraisal. Neuropsychopharmacology 2011; 36:2589-602.

35. Vaughan DA, Cleary BJ, Murphy DJ. Delivery outcomes for nulliparous women at the extremes of maternal age - a cohort study. BJOG 2014; 121:261-8.

36. Clinical Outcomes. In: KK Women's and Children's Hospital Obstetrics [online]. Available at: https://www.kkh.com.sg/AboutUs/ClinicalOutcomes/Pages/ Obstetrics.aspx. Accessed October 19, 2017.

37. Ng TP, Jin AZ, Ho R, et al. Health beliefs and help seeking for depressive and anxiety disorders among urban Singaporean adults. Psychiatr Serv 2008; 59:105-8.

38. Ng TP, Fones CS, Kua EH. Preference, need and utilization of mental health services, Singapore National Mental Health Survey. Aust N Z J Psychiatry 2003; 37:613-9.

39. Bilszta J, Ericksen J, Buist A, Milgrom J. Women's experience of postnatal depression - beliefs and attitudes as barriers to care. Aust J Adv Nurs 2010; 27:44-54.

40. Goodman JH. Women's attitudes, preferences, and perceived barriers to treatment for perinatal depression. Birth 2009; 36:60-9. 